\title{
Physician kindness as sincere benevolence
}

A

$\mathrm{n}$ attractive, physically healthy woman sits beside her physician, her generalized anxiety having brought her to the point of tears. To convey concern and offer comfort, her physician reaches out to gently touch her forearm - but he hesitates. Might this physical gesture, he wonders, reflect his need to connect, as much as - or more than - hers? Might it compromise his clinical detachment? Might the frightened woman worry that it cloaks sexual interest; shows favouritism or weakness; undermines her capacity for self-care by treating her like a child; or appears excessive or insincere?

This scenario draws attention to fear of misinterpretation as one barrier to recent calls to reconnect medicine to a culture of human kindness. ${ }^{1}$ This fear, the modern importance attached to scientific objectivity, and a risk to physicians of "compassion fatigue" can frequently overwhelm the impulse of physicians to draw closer to their patients. Although physicians spontaneously displaying emotions can help to show patients how much their physician cares, some physicians continue to fear doing just that.

As part of a movement to rehumanize medicine, Faust ${ }^{2}$ has defined kindness as a way of helping without necessarily feeling the emotional engagement required of compassion or empathy. This definition makes kindness easier to apply than compassion, and permits physicians to "act as if they care ... without taking on the stressful emotions that emanate from being compassionate or empathetic". ${ }^{2}$ For me, this perspective errs because most patients want a physician who genuinely cares - and most physicians do care.

For me, kindness is sincere benevolence that need not be self-sacrificing.

Rather than faking it, physicians' kindness toward patients must be genuine and include an element of physician self-care. Allow me to elaborate. Physician kindness opens up an opportunity for "a mutual experience of joining that results in a sensation of wholeness." ${ }^{3}$ This experience of joining matters to

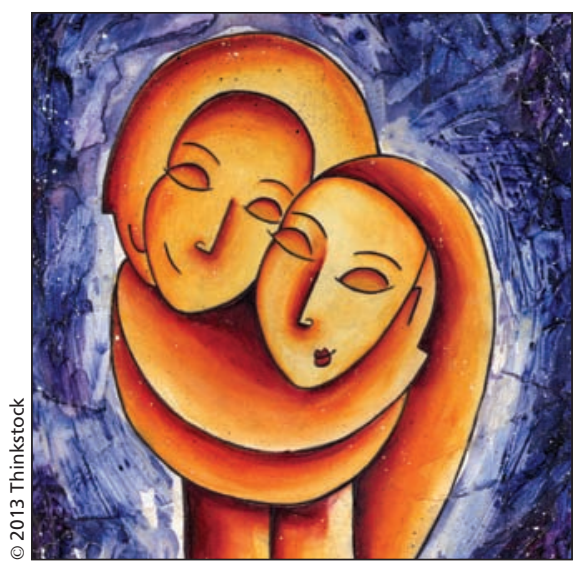

both parties because, as the African concept of "ubuntu" explains, a person's humanity depends on their connectedness to others. Kindness by the physician is ubuntu. Felt rather than chosen by the physician, it is "goodness without [conscious, reasoned] thought." Its sincerity comes from the physician instinctively accepting their own vulnerability in the act of reaching out to the patient. A consequent, shared feeling of wholeness may satisfy a mutual need for emotional connection and transform suffering into profound, positive emotions and healing.

Physician kindness is further connected to justice, which includes a principle of equality. According to this principle, physicians and patients are moral equals whose equal interests deserve equal consideration. Substitution of "equal consideration of equal interests" for the dominant principle of the "primacy of patient welfare" can be further justified by the interrelated interests of the physician and patient in doing what feels morally right and produces good consequences.

Applied to the above case example, the physician perceives the patient as having an interest in feeling understood and reassured. Responding to this interest requires the physician to have, and act appropriately on, his own emotional impulse for human connection. His interest is important for the patient's sake but also his own - because in touching her arm, he is touched. Thus, both parties have an equal interest in the interaction and in feeling connected, which helps to mitigate barriers to kindness such as fear of showing favouritism or weakness, and give moral strength to the physician.

In turn, the physician can minimize the risk of his patient reacting negatively to his touching of her arm, by finding a midpoint between too much kindness as suggested by emotions such as oversentimentality or patronizing acts such as patting the patient's arm - and too little kindness as revealed by a lack of obvious concern. Patients are able to spot this midpoint. They can discern the difference between "taught or professional kindness (a "skill"), and natural kindness — and we all know which we prefer."

Moral values such as kindness can indeed be difficult to cultivate. However, physicians should have no less capacity than others to shape their own character, for example through training and practise in moral development and in felt attitudes such as sensitivity. Physicians can then feel more secure about implementing person-centred values like kindness, and about how patients may interpret their actions. Physicians can become enabled to ground their care habitually in kindness as an experience of sincere benevolence, without letting their "heart" rule their "head."

\section{Stephen A. Buetow PhD}

Associate Professor

Department of General Practice

and Primary Health Care

University of Auckland

Auckland, New Zealand

\section{References}

1. Ballatt J, Campling P. Intelligent kindness: reforming the culture of healthcare. London (UK): Royal College of Psychiatrists; 2011.

2. Faust HS. Kindness, not compassion, in healthcare. Camb Q Healthc Ethics 2009;18:287-99.

3. Suchman AL, Matthews DA. What makes the patient-doctor relationship therapeutic? Exploring the connexional dimension of medical care. Ann Intern Med 1988;108:125-30.

4. Levinas E. In the time of the nations. Indianapolis (IN): Indiana University Press; 1994

5. Pickering WG. Kindness, prescribed and natural, in medicine. J Med Ethics 1997;23:116-8. 